\title{
Le Journal de Charles V de Lorraine comme source pour l'histoire de la reconquête de la Hongrie sur les Turcs ${ }^{1}$
}

\author{
Ferenc Tóth
}

\section{Résumé}

La personne du duc Charles V de Lorraine est étroitement liée à l'histoire de la Hongrie moderne, en particulier à cause de son rôle joué dans la reprise de la ville de Bude (1686) et dans la reconquête du pays sur les Turcs. Le but de notre étude est d'expliquer le rôle joué par Charles V dans la reconquête de la Hongrie ainsi que dans la formation d'un nouveau système de relations entre la Hongrie et l'Empire des Habsbourg. Grâce à son Journal, nous pouvons avoir une vue plus détaillée sur le parcours militaire et politique du duc de Lorraine qui était un des plus importants acteurs de la libération de la Hongrie sur les Turcs. Il nous permet de mieux comprendre ses activités spécifiques dans l'organisation et le commandement de l'armée, ainsi que son rôle politique comme chef d'armée. Enfin, nous avons analysé l'influence de son expérience en Hongrie sur son commandement dans la guerre en Europe occidentale, en particulier dans le domaine de la petite guerre.

\section{Abstract}

Charles V Leopold, duke of Lorraine (1643-1690) military and political career was strongly attached to the early modern history of Hungary, because of his important role played as commander in chief during the Great Turkish War of 1683-1699. The aim of this study is to explain the role played by Charles $V$ of Lorraine in the reconquest of Hungary and in the formation of a new relationship between Hungary and the Habsburg Empire. Thanks to this Journal, we can get a much more detailed overview about the military and political career of Charles V, Duke of Lorraine, one of the main actors of the liberation of Hungary from the Turkish occupation. It allows us to have a better understanding of the specific activities of the Duke, such as the organisation and command of the army, as well as the political role of the Duke as a military officer. Finally, we focus on the issue

1. Cette étude a été préparée avec le soutien du projet scientifique «Lendület - Sainte Couronne de Hongrie » (2012-2017) de l'Institut d'Histoire du Centre de Recherches en Sciences Humaines de l'Académie Hongroise des Sciences. 
whether he could benefit at the occidental theatre of wars from his experiences obtained in Hungary by the application of the small war.

La personne du duc Charles V de Lorraine est étroitement liée à l'histoire de la Hongrie moderne, en particulier à cause de son rôle dans la reprise de la ville de Bude (1686) et dans la reconquête du pays sur les Turcs. Dans le quartier du château de Bude plusieurs endroits gardent encore sa mémoire et le tableau historique de Gyula Benczúr (Budavár visszavétele - La reprise de Bude, 1896) le représente symboliquement, comme un héros hongrois, sur un cheval blanc. Néanmoins les recherches scientifiques sur l'activité de ce personnage sont rares en Hongrie, et cela est vrai pour les autres pays européens (France, Autriche, Allemagne). Comme beaucoup d'autres personnages d'importance internationale, Charles $\mathrm{V}$ de Lorraine reste un des grands oubliés des histoires nationales. Bien qu'il portât le titre prestigieux de duc de Lorraine et de Bar, il ne régna jamais sur ses duchés et fut surnommé « duc sans duchés ». Cela explique le manque de travaux historiques sur sa vie dont même les sources restent encore ensevelies dans différentes archives européennes.

Les documents relatifs à l'activité du duc sont dispersés en Europe. La collection la plus importante de sources est conservée à Vienne dans les archives de la Maison de Lorraine des Archives Nationales Autrichiennes (Haus-, Hof- und Staatsarchiv, Lothringisches Hausarchiv). Les journaux militaires qui font partie de cette collection furent découverts à l'occasion des commémorations historiques. En 1933, pour le $250^{\mathrm{e}}$ anniversaire du second siège turc de Vienne, Ferdinand Stöller publia un journal fort intéressant de cette série. En 1986, un journal fragmentaire du siège de Bude fut édité par les historiens hongrois ${ }^{2}$. Cet ouvrage mit en relief les documents des archives ducales liés à l'histoire hongroise. Malgré les nombreuses recherches effectuées sur les guerres turques en Hongrie, les travaux fondamentaux scientifiques manquent encore sur la vie et l'activité du duc dans les opérations militaires en Hongrie à la fin du XVII ${ }^{\mathrm{e}}$ siècle. Dans notre étude, nous souhaiterions présenter un ouvrage manuscrit comprenant l'histoire des campagnes du duc Charles V de Lorraine dont nous assurons la préparation pour une édition scientifique ${ }^{3}$.

Le Journal des campagnes de Charles $V$ de Lorraine, est un recueil de journaux des campagnes menées par le duc Charles V de Lorraine de 1683 jusqu'en 1689. Cet ouvrage fortement lié aux événements de la reconquête hongroise sous le règne de Léopold $\mathrm{I}^{\mathrm{er}}$ fut visiblement destiné à une publication à part entière. Néanmoins, il ne vit jamais le jour tel qu'il fut préparé par les collaborateurs de Charles de Lorraine. Malgré son titre clair, la question de l'identité de son auteur reste encore ambiguë. Dans notre étude, nous essayerons de présenter ce texte méconnu à travers la vie du duc de Lorraine, son auteur présumé et protagoniste principal, en nous appuyant sur ses différentes éditions et surtout en le replaçant dans le contexte des événements historiques qui furent à l'origine de sa genèse.

Le $\mathrm{XVII}^{\mathrm{e}}$ siècle correspond en Lorraine à une période de troubles et de complet affaiblissement de la puissance ducale. En 1624, un problème de succession survint avec la mort du duc Henri II sans héritier mâle. Avant de disparaître, il maria sa fille aînée, Nicole, à son cousin Charles de Vaudémont qui devait régner par la suite. Néanmoins, la

2. Lotharingiai Károly hadinaplója Buda visszafoglalásáról 1686 (Journal militaire de Charles de Lorraine sur la reprise de Bude), Károly Mollay (éd.), Budapest, HIM, 1986.

3. L'édition critique du texte du Journal des campagnes de Charles de Lorraine sera publiée par nos soins aux éditions Honoré Champion, probablement en 2015 ou 2016. 
découverte du testament du duc René $\mathrm{II}^{4}$ sema le trouble, car il affirmait que la couronne ducale ne pouvait se transmettre que de mâle en mâle. Il en résulta que le successeur légitime était François de Vaudémont, le père de l'époux de Nicole, qui renonça aussitôt au pouvoir en faveur de son fils qui commença à gouverner seul sous le nom de Charles IV. Son comportement imprévisible et son attachement à l'Empereur provoquèrent l'hostilité de la France dont les troupes occupèrent en 1633 les États de Charles IV, qui abdiqua au profit de son frère, et participa à la guerre de Trente Ans dans l'armée impériale. En 1641, il réussit à récupérer ses États par le traité de Saint-Germain-en-Laye, mais il dut accepter le protectorat français et renoncer à l'alliance impériale. Peu après, il recommença le combat contre la France jusqu'à la fin de la guerre. Plus tard, profitant des troubles de la Fronde, il reprit la guerre et menaça même Paris en 1652. Après la fin de la Fronde, le duc Charles séjourna aux Pays-Bas espagnols où il s'attira la méfiance du gouverneur Fuenseldagna qui le fit arrêter et transférer à l'Alcazar de Tolède dont il ne sortit qu'en $1659^{5}$, mais il ne récupéra ses États qu'en 1661 par le traité de Vincennes. Son caractère belliqueux ne laissa pas de paix à la France de Louis XIV qui finit par occuper de nouveau le duché en 1670 puis le garder jusqu'au traité de Ryswick (1697). Après la mort de Charles IV, survenue en 1675, son neveu Charles-Léopold-Nicolas-Sixte de Lorraine lui succéda officiellement comme duc titulaire de Lorraine sous le nom de Charles $\mathrm{V}^{6}$.

Charles V de Lorraine était le fils de Nicolas-François de Vaudémont et Claude de Lorraine qui s'enfuirent en 1634 en Italie, puis à Vienne. Comme la Lorraine était occupée par les troupes françaises, le jeune homme vécut à Vienne à la cour de l'Empereur Léopold $\mathrm{I}^{\mathrm{er}}$ qui lui donna une formation digne d'un futur souverain. Ensuite, il partit avec son père à Paris où il passa quelques années dans la cour royale. Peu avant sa mort, le duc Charles IV conclut un traité avec Louis XIV, le traité dit de Montmartre (le 6 février 1662), par lequel il lui remettait ses duchés tout en s'en réservant la souveraineté nominale jusqu'à sa mort. Profondément déçu du comportement de son oncle et du roi de France, le jour suivant, le jeune Charles de Lorraine quitta définitivement Paris et après quelques tentatives avortées de regagner ses États, il s'engagea au service de l'Empereur. La guerre s'alluma alors entre la monarchie des Habsbourg et l'Empire ottoman ce qui donna au jeune prince lorrain de 21 ans une formidable occasion de se distinguer comme ses illustres ancêtres dans le combat contre les infidèles ${ }^{7}$. Il participa à la bataille de Saint-Gotthard le $1^{\text {er }}$ août 1664 , où il s'illustra. En 1675, il fut nommé généralissime des armées impériales et prit aussi le titre de duc de Lorraine et de Bar, car son oncle était mort la même année. Toutes les puissances européennes le reconnurent comme tel, à l'exception de la France, qui occupait les duchés à cette époque, d'où son surnom. En septembre 1683, il contribua à la levée du siège de Vienne avec le roi Jean III Sobieski. Ensuite, il mena plusieurs campagnes en Hongrie occupée par les Turcs et se distingua dans la reconquête de ce pays. En 1686, il réussit à reprendre la ville de Bude, ouvrant ainsi une campagne rapide et victorieuse qui chassa les Turcs de la Slavonie et de la Transylvanie. En 1687, le duc Charles V assista à Presbourg au couronnement de l'archiduc Joseph comme roi de Hongrie. Le duc de

4. René II (1470-1508).

5. Les circonstances semblant favorables, Charles IV se livra à un double, voire un triple jeu, négociant à la fois avec Mazarin, avec les frondeurs, avec les Espagnols. Le résultat ne tarda pas et Charles IV perdit toute crédibilité, il fut arrêté après son retour à Bruxelles en 1654, et rendu responsable de tous les échecs de l'Espagne. Voir sur ce sujet : Nicolas Du Bois de Riocour, Histoire de l'emprisonnement de Charles IV, duc de Lorraine, détenu par les Espagnols dans le château de Tolède, Cologne, P. Marteau, 1688.

6. Henry Bogdan, La Lorraine des ducs. Sept siècles d'histoire, Paris, Perrin, 2005, p. 162-188.

7. Stéphane Gaber, Et Charles V arrêta la marche des Turcs... Un Lorrain sauveur de l'Occident chrétien, Nancy, Presses Universitaires de Nancy, 1986, p. 19-26. 
Lorraine y suivit la cour avant de regagner Innsbruck, chef-lieu de son gouvernement du Tyrol. Il avait fait une visite à l'empereur Léopold pour prendre congé de lui. L'entrevue se tint le 29 décembre. Le duc remit à l'Empereur un mémoire manuscrit dont il était l'auteur avec la recommandation de le lire attentivement, de le faire lire à Joseph et aux membres de la famille impériale. Le mémoire était intitulé : Testament politique de Charles, duc de Lorraine et de Bar, en faveur du roi de Hongrie et de ses successeurs arrivant à l'Empire.

La lecture même superficielle du Journal nous permet de distinguer son auteur de la personne de Charles V de Lorraine. Les événements de la vie du duc sont racontés à la troisième personne du singulier, tandis que le narrateur apparaît de temps à autre comme témoin ou chroniqueur de ceux-là. Bien que l'identité du narrateur ne pas soit révélée dans le texte, les recherches philologiques ont, depuis longtemps, attribué ce travail à un certain M. Le Bègue ${ }^{8}$. La famille Le Bègue de Germiny était bien connue à cette période en Lorraine. Elle était issue de Vian Pistor, un diplomate du duc Charles III, qui fut anobli en 1596. En 1599, il épousa Anne Serre dont il eut un fils, Charles (1599-1667), seigneur de Germiny et de Chanteraine, conseiller des ducs François et Charles IV de Lorraine. Charles Le Bègue épousa en 1630 Marguerite de Rutant, dame de Chantereine et de Dompsévrin. Ils eurent de leur mariage quatorze enfants, dont huit moururent en bas âge. Des survivants, c'est incontestablement l'aîné, François Le Bègue de Germiny, qui fit la plus belle carrière. Le chevalier François, né en 1639, fut nommé grand doyen de l'église primatiale de Nancy en 1669, ensuite doyen de l'église de Saint-Dié et grand vicaire du primat. Il devint plus tard abbé commanditaire de l'abbaye de Bouzonville, ordre de Saint-Benoît, par provisions expédiées à Francfort-sur-le-Main, le 20 septembre 1673. Après avoir servi le duc Charles IV de Lorraine, l'abbé Le Bègue fut sincèrement attaché à la personne et à la fortune du duc Charles V, suivit ce prince en Allemagne, et recueillit avec soin tout ce qui fut dit et écrit de plus important sur la guerre et la politique ${ }^{9}$.

Chargé par ce même prince de négociations secrètes en Espagne et envoyé plus tard à Cologne pour conférer avec l'évêque de Strasbourg au sujet du rétablissement de son souverain en Lorraine, il eut assez de courage et de fermeté pour repousser les offres de la France, comme contraires à l'honneur et à la liberté de celui qu'il représentait. En 1690, l'Empereur le nomma gouverneur du jeune duc Léopold. François Le Bègue accompagna son illustre élève qui allait faire ses premières armes contre les Turcs, dans les rangs impériaux, en 1694 où il se distingua dans la bataille de Temesvár ${ }^{10}$. Le duc Léopold appelé dans ses États à la suite du traité de Ryswick chargea l'abbé Le Bègue avec le comte de Carlinford et le baron de Canon d'administrer la Lorraine avant qu'il n'en prît possession définitive. L'abbé Le Bègue rentra seul à Nancy le 8 février 1698. Cet homme d'État, après avoir eu pendant sa vie une grande part dans le gouvernement de la Lorraine, en qualité de garde des Sceaux et premier ministre des ducs Charles IV, Charles V et Léopold, mourut à Nancy le 19 juillet 1699 et fut inhumé dans l'église primatiale ${ }^{11}$. François Le Bègue fut un homme de confiance du duc Charles V ainsi qu'un des exécuteurs de ses testaments. Il laissa sur la vie de ce prince des mémoires manuscrits qui ont servi à Dom Calmet pour composer

8. Le texte partiellement reproduit dans l'ouvrage de Dom Calmet (Histoire ecclésiastique et civile de Lorraine) était attribué dans les notes infrapaginales aux Mémoires mss. de M. Le Bègue. Voir infra.

9. Alain Petiot, Les Lorrains et l'Empire. Dictionnaire biographique des Lorrains et de leurs descendants au service des Habsbourg de la Maison d'Autriche, Versailles, Ed. Mémoire \& Documents, 2005, p. 307.

10. Henri Baumont, Études sur le règne de Léopold, duc de Lorraine et de Bar (1697-1729), Paris, BergerLevrault, 1894, p. 33.

11. François-Alexandre Aubert de La Chesnaye Des Bois (éd.), Dictionnaire de la noblesse : contenant les généalogies, l'histoire et la chronologie des familles nobles de France, t. 2, Paris, Schlesinger frères, 1863, p. $827-828$. 
son Histoire de Lorraine. Après le travail monumental de l'érudit lorrain, les manuscrits de l'abbé Le Bègue furent redécouverts par les historiens autrichiens, notamment Ferdinand Stöller qui publia en 1933 un manuscrit tiré des archives de la Maison de Lorraine sur l'histoire du secours de Vienne en 1683. Dans l'introduction de sa publication de sources, il présenta même une brève biographie de l'abbé de Bouzonville ${ }^{12}$.

D’après la documentation attachée aux cartons $\mathrm{n}^{\circ}$ 50-51 des archives de la Maison de Lorraine des Archives Nationales Autrichiennes (Haus-, Hof- und Staatsarchiv, Lothringisches Hausarchiv) nous pouvons découvrir quelques aspects des méthodes de travail de l'auteur. Outre le texte relu et corrigé, nous avons accès aux différents journaux manuscrits qui constituaient la base du travail fini. Dans les versions antérieures, nous pouvons trouver des indications ainsi que des notes et messages qui reflètent les méthodes utilisées par l'abbé Le Bègue. Comme il suivait le plus souvent la cour du duc de Lorraine, il tenait un journal qu'il retravaillait avec soin à la fin de l'année pour présenter l'histoire de la campagne à son maître. Par ailleurs, il ajoutait d'autres documents, des récits, des lettres et des pièces justificatives qu'il intégrait également dans son histoire. En sa capacité de témoin oculaire des événements, il devint en quelque sorte le chroniqueur des grands faits d'armes de Charles V de Lorraine. L'idée de composer un journal des campagnes vint très probablement à la fin de l'année 1683, lorsque la libération de Vienne fit du duc de Lorraine un héros européen. Le succès du rapport détaillé de la campagne de 1683 favorisa l'écriture de la campagne suivante, moins heureuse que la précédente, mais non moins intéressante pour le chroniqueur témoin. Une lettre adressée au duc et insérée dans le dossier du récit de la campagne de 1684 nous explique ainsi les motifs de sa création : «L'honneur que V. Excellence m'a fait de me tesmoigner qu'elle aggréoit ma relation de la campagne passée, m'oblige à Luy envoyer présentement le detail de celle que nous venons d'achever pour satisfaire au désir qu'elle a d'en estre esclairée ${ }^{13}$. » L'année suivante, une autre lettre annonça la suite de l'ouvrage, depuis la fin de la partie précédente, où l'auteur évoque déjà les ordres spécifiques donnés par le duc concernant la rédaction du Journal : «Pour obéir aux ordres que V. Excellence me donne de Luy faire le détail de la campagne que nous venons d'achever je reprendray la suitte de ma derniere relation et je commenceray celle cy par l'arrivée du Duc de Lorraine à la Cour au mois de décembre de l'an $1684^{14}$. » En 1686, l'année de la reprise de Bude, Le Bègue présenta ainsi à son souverain son travail : «L'interest que V. Excellence prend aux sucez de cette guerre et le plaisir qu'Elle a d'en apprendre. Les principalles actions m'obligent de luy envoyer la relation de la campagne que nous venons d'achever ${ }^{15}$. » L'année 1687, celles des grandes victoires, fut introduite par le chroniqueur toujours avec le même enthousiasme de rendre immortels les faits d'armes de son maître : «La campagne que nous venons d'achever estant une des plus grandes que nous ayions fait et une suitte glorieuse des victoires de Sa Majesté Imperiale, j'ay crû que V. Excellence seroit bien aise que je luy en fis le détail avec la mesme exactitude que j'ay eu dans mes relations precedentes ${ }^{16}$. » Comme l'auteur insista sur le principe de l'exactitude, sa méthode scientifique commençait à s'approcher de celles des historiens. La campagne suivante fut différente des précédentes, car François

12. Ferdinand Stöller, « Neue Quellen zur Geschichte des Türkenjahres 1683 », Mitteilungen des österreichischen Instituts für Geschichtsforschung, Ergänzungsband XIII, H. 1, Innsbruck, 1933, p. 3.

13. ÖStA (Österreichisches StaatsArchiv), Haus-, Hof- und Staatsarchiv, Hausarchiv, Lothringisches Hausarchiv Kt. 51, Relation contenant les actions de l'an 84.

14. Idem, Relation contenant le detail des actions de la campagne de 85 .

15. Idem, fol. 46.

16. Idem, fol. 69. 
Le Bègue n’y était pas présent. Néanmoins, notre historien dépouilla les archives pour reconstruire les événements des opérations de 1688 :

Quoy que j'ay peine d'escrire les actions de la campagne derniere parce que ne les ayant pas veües, je n'en peux pas faire le détail avec la mesme exactitude que j'ay eu dans mes relations précédentes. Neantmoins comme les desseins en ont esté extrêmement hardis, les sucez heureux, et qu'on y voit mieux qu'en aucun autre, qu'une armée victorieuse peut tout entreprendre sur un Ennemy vaincu, je me trouve obligé de continuer une aussy belle suitte des glorieux progrez des armées de l'Empereur, et pour en faire une narration fidelle, j' ay fait rechercher les actes des délibérations des Conseils de Guerre avec touttes les lettres que le Duc de Lorraine a receües des principaux generaux de l'armée dont voicy le recueil ${ }^{17}$.

Le succès de la reconstruction du passé non vécu fut tellement rassurant que l'auteur continua même après la guerre son travail de mémorialiste à partir des documents d'archives. Après la rédaction de l'histoire des campagnes de Hongrie, François Le Bègue réunit d'autres récits de campagnes qui, conformément aux méthodes d'autres mémorialistes de son époque, pouvaient servir de sources et aux futurs grands historiens. Cette collection, mémoires des campagnes militaires du duc à partir de 1670 jusqu'en 1689, constituait une précieuse matière première pour une histoire des ducs de Lorraine, comme le remarqua l'abbé Le Bègue dans l'introduction de ses Mémoires des campagnes:

Ayant trouvé à propos de ramasser quelques memoires des dernières campagnes de Hongrie, j'ay voulu y adjouter ce que j'ay retrouvé de celle de l'Empire affin de trouver un seul volume les principales actions de ces guerres. Il n'y a nul ordre, il $\mathrm{y}$ a beaucoup de fautes de stile et d'escriture et ce n'est qu'un ramas de diverses pieces qui n'ont pas mesme esté relues et qui estant séparées se fussent perdue mais la verité y est toutte pure et ainsy elles peuvent servir dans la suitte à relever la gloire de nos souverains ne perdant pas l'esperance de pouvoir jouyr de quelque repos et m'occuper un jour à revoir touts ces fragments pour les mettre ne quelque ordre ${ }^{18}$.

Le Journal des campagnes de Charles $V$ de Lorraine fut exploité pour la première fois par le savant historien Dom Calmet. Augustin Calmet fut un exégète et érudit lorrain, savant bénédictin de la Congrégation de Saint-Vanne et Saint-Hydulphe. Originaire de la région de Commercy, il entra dans la vie religieuse au prieuré du Breuil. Il fit ses études à l'université de Pont-à-Mousson où il suivit les cours de rhétorique du père jésuite Ignace L'Aubrussel, le futur confesseur de la reine d'Espagne. Après ses études, il entra dans l'ordre bénédictin où il prononça ses vœux en 1689 . Il continua ensuite ses études de philosophie et de théologie à Toul et à Munster. Il fut ordonné prêtre en 1696. Il fut chargé d'expliquer les saintes Écritures, ce qui lui fit une réputation en matière d'exégèse et d'histoire biblique. Il publia entre 1706 et 1716 ses Commentaires sur tous les livres de l'Ancien et du Nouveau Testament qui devinrent rapidement un ouvrage de référence. Il fit paraître également un Dictionnaire historique et critique de la Bible en 1720 à Paris qui fut traduit en latin, allemand et anglais. Dom Calmet vécut successivement à Munster, à Moyenmoûtier, à Paris, au couvent des Blancs-Manteaux, à Saint-Mihiel où il termina

17. Idem, fol. 91.

18. ÖStA, Haus-, Hof- und Staatsarchiv, Hausarchiv, Lothringisches Hausarchiv Kt. 50, Memoires des campagnes et des actions de guerre depuis 1670 jusqu'en 1689, fol. 11. 
les 26 volumes de son Commentaire sur l'Ancien et le Nouveau Testament, au prieuré de Lay-Saint-Christophe, à l'abbaye Saint-Léopold de Nancy. C'est là qu'il publia la première édition de sa monumentale Histoire ecclésiastique et civile de Lorraine, ouvrage qui lui avait été commandé par le duc Léopold. Ce travail fut confié d'abord à l'abbé Charles-Hyacinthe Hugo qui fut ensuite écarté à cause de ses idées politiques ${ }^{19}$. Voltaire fit sa connaissance pendant ses séjours lorrains et, lorsqu'il travaillait sur son Histoire universelle, il passait des semaines dans la bibliothèque de Senones dont Dom Calmet fut l'abbé. Il écrivit ainsi dans sa lettre du 13 juin 1754 à Marie-Louise Denis : « Je m'occupe à l'Histoire dans une bibliothèque immense. Les moines me cherchent les pages, les lignes, les citations que je demande. Dom Calmet à l'âge de quatre-vingt-trois ans, monte en haut d'une échelle qui fait trembler, et qui tremble, et il me déterre de vieux bouquins ${ }^{20}$. »

Dom Calmet, et ses collaborateurs certainement nombreux, utilisèrent abondamment le texte du Journal qui était en quelque sorte un fil conducteur des récits des campagnes du duc Charles V. Le texte fut parfois à peine modifié lorsqu'ils insérèrent dans l'ouvrage monumental de l'Histoire ecclésiastique et civile de Lorraine. Ces modifications sont surtout des rectifications orthographiques et stylistiques qui enlèvent les tournures parfois lourdes et très influencées par le dialecte lorrain de l'auteur. Le résultat est un texte nettoyé de fautes de vocabulaires et d'idiotismes archaïques. Néanmoins, le travail de compilation de Dom Calmet avait gardé le contenu précieux du Journal qui nous rendait un très grand service lors du déchiffrement du manuscrit. De temps à autre, le rédacteur de cet ouvrage mit des références des sources qui nous permettaient, en partie, d'identifier l'auteur du Journal. Dans les renvois, le titre exact du manuscrit n'apparaît jamais exactement, le compilateur se contenta d'y mettre la référence suivante : Mémoires de M. Le Bègue. Le mot «mémoires » y fut employé dans le sens exact du genre littéraire et historique apparu aux $\mathrm{XVI}^{\mathrm{e}}$ et $\mathrm{XVII}{ }^{\mathrm{e}}$ siècles. À cette époque, on attendait le grand historien, l'équivalent moderne d'un Tite-Live, capable de composer pour la postérité le récit glorieux des règnes des souverains, grand historien qui serait nécessairement proche du pouvoir. De manière en apparence très modeste, les premiers mémorialistes, dès le XVII ${ }^{\mathrm{e}}$ siècle, se présentaient comme les collecteurs d'informations préparatoires au travail futur du grand historien qui devait faire la synthèse panoramique et la belle rhétorique. Les mémoires représentaient le témoignage parcellaire, sans ambition de style, mais garanti par l'expérience du témoin. D'une manière rétrospective, le Journal de Charles V de Lorraine, rédigé par François Le Bègue, fut considéré comme modèle du genre et rebaptisé Mémoires mss. de M. Le Bègue dans l'ouvrage de Dom Calmet ${ }^{21}$.

Mais comment ce manuscrit aujourd'hui conservé à Vienne pouvait-il servir de source pour un historien lorrain du début du XVIII ${ }^{\mathrm{e}}$ siècle ? Pour la bonne compréhension des choses, il convient de rappeler une brève histoire des archives ducales lorraines. À l'origine, ces archives, le fameux Trésor des chartes de Lorraine, se trouvaient à Nancy à la collégiale

19. Voir récemment sur l'activité historiographique de Dom Calmet : Cédric Andriot, « Dom Calmet : une œuvre à l'épreuve du temps », dans Dom Augustin Calmet : un itinéraire intellectuel, dir. Philippe Martin et Fabienne Henryot, Paris, 2008, p. 49-65 ; Thomas Nicklas, « Affirmer, nier, ou demeurer dans le doute. » Lothringens Geschichtsschreiber Dom Calmet in der historiographische Grauzone », dans Glaubensformen zwischen Volk und Eliten. Frühneuzeitliche Praktiken und Diskurse zwischen Frankreich und dem Heiligen Römischen Reich, dir. Thomas Nicklas, Universitätsverlag Halle-Wittemberg, 2011, p. 43-57.

20. Cité par Béla Köpeczi, « Les vampires de Hongrie : un scandale des Lumières », dans Béla Köpeczi, Hongrois et Français. De Louis XIV à la Révolution française, dir. Béla Köpeczi, Budapest, Corvina, 1983, p. 315 .

21. Voir sur le genre des mémoires politiques à l'époque moderne : Mémoires d'État et culture politique en France (XVI $I^{e}$ XIX $X^{e}$ siècles), dir. Jean Garapon, Nantes, C. Defaut, 2007. 
Saint-Georges. Au XVI ${ }^{\mathrm{e}}$ siècle, les archives passées sous le contrôle de la chambre des comptes furent conservées dans une tour octogonale du palais ducal. Lorsque les troupes françaises envahirent la Lorraine en 1633, elles furent transférées à la forteresse de La Mothe, et après la chute de celle-ci, directement à Paris. Dans la capitale française, elles furent conservées à la Sainte-Chapelle d'où elles retournèrent amoindries à Nancy en 1665. Lors de la seconde occupation française de la Lorraine, en 1670, le Trésor des chartes fut transféré à Metz, d'où il revint de nouveau amoindri en 1698-1699, conformément au traité de Ryswick (1697). Les archives retrouvèrent alors leur ancienne tour octogonale et elles y restèrent jusqu'à l'arrivée de Stanislas Leszczynski. En 1736, l'article 16 du traité de Vienne prévoyait la cession à Stanislas des documents concernant les duchés, mais les papiers de la Maison de Lorraine devaient rester au duc François III de Lorraine. Ce dernier donna alors ordre de faire partir 204 paquets du Trésor des chartes. Les archives furent transportées par bateaux aux Pays-Bas autrichiens d'où elles furent conduites par mer en Italie, parce que François III devint grand-duc de Toscane, et une partie directement à Vienne. Après la rédaction d'un inventaire détaillé des documents à Florence par le secrétaire Thierry, les archives lorraines furent bientôt emportées à Vienne où elles furent réunies aux archives impériales sous le règne de Joseph II. Complétées par les autres archives de la famille ducale, les archives de la Lorraine finirent par être intégrées, après la chute de la Monarchie austro-hongroise, dans les archives nationales autrichiennes. Depuis 1925, le fonds lorrain de Haus-, Hof- und Staatsarchiv est propriété fédérale ${ }^{22}$. Toutes ces circonstances expliquent comment Dom Calmet pouvait bénéficier encore au début du XVIII ${ }^{\mathrm{e}}$ siècle à Nancy des sources archivistiques qui sont aujourd'hui conservées à Vienne. Nous ne savons pas exactement à quel moment les manuscrits de François Le Bègue furent intégrés dans les archives ducales. D'après le témoignage de Dom Calmet, lors de la rédaction de son ouvrage historique, ils étaient encore dans la possession de la famille. Dans son ouvrage intitulé Bibliothèque lorraine publié en 1751 à Nancy, l'érudit bénédictin caractérisait ainsi l'œuvre de François Le Bègue :

Begue (François le) Doyen de S. Diey, a écrit des Mémoires sur la vie du Duc Charles V. qui sont demeurés Manuscrits. François le Begue suivit le Duc Charles V. en Allemagne, \& a recueilli \& conservé avec beaucoup de soin tout ce qui se disoit \& s'écrivoit dans les Nouvelles publiques, \& dans les Lettres particulières adressées à Son Altesse, touchant les affaires de Guerre \& de Politique. Il en a composé plusieurs Volumes Manuscrits, qui nous ont été communiqués, \& dont nous avons fait usage dans notre Histoire de Lorraine. M. le Begue de Chanterene son frère en étoit alors dépositaire ; ils sont à présent dans sa famille. M. le Begue étoit aussi Grand-Doyen de la Primatiale de Nancy. Il revint en Lorraine avec le Duc Leopold I. \& eut beaucoup de part au Gouvernement de la Lorraine, jusqu'à sa mort arrivée en $1699^{23}$.

Le journal des campagnes du duc de Lorraine comporte beaucoup d'informations sur les événements du début de la guerre de reconquête de la Hongrie. Le texte se présente avant tout comme un descriptif des opérations militaires où chaque campagne est présentée dans l'ordre chronologique et d'une manière bien structurée. Les préparatifs des campagnes

22. Hubert Collin, «Les archives de la Maison de Lorraine à Vienne », dans Les Habsbourg et la Lorraine (Actes du colloque international organisé par les Universités de Nancy II et Strasbourg III, 22, 23, 24 mai 1987), dir. Jean-Paul Bled, Eugène Faucher et René Taveneaux, Nancy, Presses Universitaires de Nancy, 1988, p. 29-33.

23. Dom Augustin Dom Calmet, Bibliothèque lorraine, ou Histoire des hommes illustres qui ont fleuri en Lorraine, dans les Trois Évêchés, dans l'archevêché de Trèves, dans le duché de Luxembourg, etc., Nancy, A. Leseure, 1751, p. 99. 
y sont minutieusement décrits, avec des tableaux détaillés contenant les effectifs des forces en présence. Même s'il ne s'agit que de chiffres sur le papier, ces données fournissent des éléments statistiques très précieux pour l'évaluation de la quantité et de la qualité des armées impériales participant aux campagnes. On y trouve suffisamment d'informations concernant les objectifs des campagnes et l'auteur ne nous cache point les âpres débats qui précédaient les décisions. On y voit clairement les différentes factions du Conseil aulique de la guerre de Vienne, ainsi que le rôle de l'Empereur dans le processus de la prise des décisions. Les bilans des campagnes nous permettent de voir à la fois les résultats militaires et leurs conséquences politiques souvent commentées d'une manière personnelle.

Autre apport considérable à l'histoire hongroise de cette source, la présentation de la guerre civile en Hongrie qui fut à l'origine de la guerre turque de 1683-1699. En effet, à partir de 1681, Éméric Thököly s'appuya de plus en plus sur l'Empire ottoman et entreprit la réalisation de son propre État, et la Hongrie se trouvait bientôt coupée non plus en trois, mais en quatre ${ }^{24}$ ! Comme allié du Grand Seigneur, Thököly dut participer à la campagne ottomane de 1683 contre Vienne. Sa popularité baissa alors dans l'Europe chrétienne, car même le roi de Pologne, sympathisant depuis longtemps avec les Mécontents hongrois, contribua à la libération de la capitale impériale. Évidemment, Thököly surestima la capacité de l'armée ottomane, qui fut battue devant les murs de Vienne. L'offensive des armées alliées après la victoire de Vienne se tourna vers l'est et Thököly dut reculer tandis que son État éphémère sombrait dans l'anarchie complète ${ }^{25}$. Après la prise de la forteresse d'Érsekújvár ${ }^{26}$, l'armée impériale occupa l'une après l'autre les places fortes fidèles à Thököly : le 11 septembre 1685 la ville d'Eperjes ${ }^{27}$, le 29 septembre celle de Tokaj et les châteaux moins importants ouvrirent leurs portes. Thököly se retira progressivement du territoire du Royaume de Hongrie. Il essaya encore de retrouver la bienveillance des rois européens en leur proposant son aide contre les Turcs, mais ces tentatives semblèrent trop tardives... Cependant, les Turcs voulaient également se servir de sa personne lors des négociations. De cette façon, le pacha de Nagyvárad ${ }^{28}$ l'invita dans sa résidence et l'emprisonna le 15 octobre $1685 .^{29}$ L'État éphémère de Thököly se décomposa rapidement après l'enlèvement de son prince. Très rapidement, toutes ses forteresses se rendirent, à l'exception de celle de Munkács défendue par sa femme, Hélène Zrínyi. Le journal des campagnes de Charles de Lorraine suit précisément le déclin du mouvement de Thököly et décrit le retour de ses partisans au service de l'empereur. Le rôle joué par le duc de Lorraine dans la pacification de la Hongrie fut primordial.

Les progrès des forces impériales changèrent radicalement la situation politique en Hongrie. Longtemps hostiles à la politique de Léopold $\mathrm{I}^{\mathrm{er}}$, les Hongrois étaient alors très

24. Stéphane Gaber, Et Charles V..., op. cit., p. 76.

25. Jean Bérenger, Finances et absolutisme autrichien dans la seconde moitié du XVII e siècle, Paris, Publications de la Sorbonne, 1975, p. 74-77.

26. Aujourd'hui Nové Zámky en Slovaquie.

27. Aujourd'hui Presov en Slovaquie.

28. Aujourd'hui Oradea en Roumanie.

29. Son biographe français de l'époque, Jean Le Clerc, immortalise ainsi la scène de sa capture : «Le Bassa leur vingt au-devant et les fit entrer dans la ville au bruit de toute l'Artillerie. L'Armée campa dans les environs, pendant que les Officiers furent régalés par le Bassa qui leur faisoit très bon visage. Mais à la sortie de table, comme Tekeli s'attendoit à entrer dans une conference particuliere, quelques Janissaires entrèrent dans la Chambre, avec des chaines dont ils le chargerent, par le commandement du Bassa, qui avoit reçu, disoit-il, cet ordre de la Porte. » Jean Le Clerc, Histoire d'Emeric comte de Tekeli ou mémoires pour servir à sa vie où l'on voit ce qui s'est passé de plus considérable en Hongrie depuis sa naissance jusques à present, Cologne, 1694, p. 173 . 
impressionnés par l'avancée des troupes impériales dans le pays, la défaite de Thököly et des Mécontents en Transylvanie, et par les soulèvements dans l'Empire ottoman. Le moment parut idéal à la Cour de Vienne pour conclure un compromis avec les ordres de Hongrie. Léopold $\mathrm{I}^{\mathrm{er}}$ poussa alors les préparatifs pour la convocation de la Diète à Presbourg ${ }^{30}$ où elle se réunit à la fin de l'année 1687. L'enjeu des négociations se révélait majeur : outre le couronnement de l'archiduc Joseph, il s'agissait de reconnaitre l'hérédité de la dynastie des Habsbourg sur le trône de Hongrie qui était encore, de droit, une monarchie élective. Les débats furent souvent houleux et vifs à cause des représentations des ordres sur le poids des contributions militaires et les excès de la soldatesque impériale dans le pays. Finalement, sous la pression des magnats catholiques et des représentants du clergé, les ordres acceptèrent le principe de l'hérédité de la Maison d'Autriche en Hongrie qui fut aussitôt inscrit dans la loi de 1687 et bientôt promulguée le 25 janvier 1688. Cette loi institua une modification radicale de la constitution du royaume de Hongrie. D'autre part, la Diète abolit la clause de la Bulle d'or d'André II de 1222 qui autorisait la résistance armée et légalisait les révoltes nobiliaires ${ }^{31}$. C'est avec beaucoup de raison que le duc de Lorraine, dans son Journal, qualifiait cet événement de révolution :

Je ne puis pourtant en finir le récit, sans adjouter deux grandes révolutions qui en furent les suites heureuses. La premiere fut dans le changement de gouvernement du Royaume de Hongrie qui d'électif fut declaré héréditaire. L'Empereur qui avoit de grandes prétentions sur cette courone depuis plus de deux siècles que les Princes de son sang l'avoient possédée jugea qu'il ne pouvoit rencontrer une conjoncture plus propre à les faire décidera à son avantage que celles de tant de victoires dont Dieu benissoit continuellement, ses armes et dans ce dessein Sa Majesté resolut d'en convoquer les Estats à Presbourg à la fin de la campagne et de leur faire témoigner que pour couper la racine des divisions et des guerres intestines qui avoient cy devant troublé le repos du Royaume, Elle avoit jugé necessaire de leur faire voir les prétentions legitimes qu'Elle avoit à la succession de leur Couronne, et de les prier de se déporter en faveur des Princes de Sa Maison du privilège qu'ils pretendoient avoir d'élire un Roy, les faisant asseurer en mesme temps de la résolution que $\mathrm{Sa}$ Majesté avoit prise de les remettre dans leurs autres privileges, et de les faire joüyr de tous les avantages qui doivent faire le repos et la tranquillité des monarchies ${ }^{32}$.

En 1687, le duc Charles V assista à Presbourg au couronnement de l'archiduc Joseph comme roi de Hongrie. Le duc de Lorraine y suivit la cour avant de regagner Innsbruck, chef-lieu de son gouvernement du Tyrol. Il avait fait une visite à l'Empereur Léopold pour prendre congé de lui. L'entrevue se tint le 29 décembre. Le duc remit alors à l'empereur son testament politique, un mémoire manuscrit à l'attention du roi de Hongrie et ses successeurs. Cet ouvrage est un recueil de pensées et de recommandations pour le gouvernement intérieur et extérieur de la Monarchie des Habsbourg qui fut même publié, dans des conditions obscures, en 1697 à Leipzig et à Cologne. En effet, la politique extérieure impériale du début du XVIII ${ }^{\mathrm{e}}$ siècle ainsi que le gouvernement du duc François III,

30. Aujourd'hui Bratislava en Slovaquie.

31. Jean Bérenger, La Hongrie des Habsbourg, t. I, De 1526 à 1790, Rennes, Presses Universitaires de Rennes, 2010, p. 159-165.

32. ÖStA, Haus-, Hof- und Staatsarchiv, Hausarchiv, Lothringisches Hausarchiv, Kt. 51, $\mathrm{n}^{\circ}$ 3, Beschreibung der Feldzüge Herzogs Karl V. von Lothringen (1683-1689), fol. 232. 
l'Empereur François $\mathrm{I}^{\mathrm{er}}$ descendant direct de Charles V de Lorraine, et sa femme MarieThérèse d'Autriche semblaient bien adopter certains principes du Testament. Le lien entre son texte et les décisions impériales reste néanmoins flou et caché. Au-delà des prophéties politiques, économiques, diplomatiques et militaires, ce texte connut surtout une vogue dans l'historiographie française qui en fit parfois un texte fondamental de la politique des Habsbourg ${ }^{33}$. Par ailleurs, les réformes proposées par le duc de Lorraine peuvent être rapprochées de celles d'un document célèbre de l'époque : le plan de réorganisation de la Hongrie (Das Einrichtungswerk des Königreichs Ungarn) du cardinal Léopold Kollonich ${ }^{34}$.

La pacification de la principauté de Transylvanie fut une réussite à la fois militaire et diplomatique. L'apparition de l'armée impériale changea complètement la situation dans cette principauté. Avec un bon sens de la négociation, le duc de Lorraine occupa les points stratégiques du pays tout en concluant des accords de compromis avec l'élite transylvaine. La Journal reproduit même les principaux documents du compromis avec le prince Apafi. La pacification de la Transylvanie fut tellement bien réussie que la Cour de Vienne voulut même offrir le gouvernement de cette principauté à Charles de Lorraine. Peut-être cela fut-il proposé en compensation pour ses duchés perdus, qui étaient d'ailleurs des obstacles permanents dans les négociations avec la France, ou bien les ministres de Vienne voulaient-ils tout simplement fixer le duc dans cette contrée afin d'y introduire un gouvernement impérial et renforcer frontières militaires avec l'Empire ottoman, comme nous en témoigne, un document résumant les événements de l'année 1687 dans les archives de la Maison de Lorraine :

Mais à la cour on fit courir le bruict que l'Empereur donneroit au Duc la Transilvanie, le Comte Ferari le creut, et jugeoit qu'il estoit plus avantageuse à Sa Majesté de la donner à S. A. ; que de la conserver soy mesme, il avoit mesme fait un long escrit où il en deduisoit toutes les raisons, le cardinal Colnitch estoit de mesme sentiment, il escrivit au Duc que l'Empereur vouloit la Luy donner, le comte de Stratman parloit moins nettement, il escrivit pour tant à S. A. que l'Empereur l'envoyant en Transilvanie, c'estoit dans le dessein qu'il travailla pour luy mesme, en faisant la conqueste de cette Province, et je crois que si avant qu'elle eut este conquise le Duc l'eut demande à l'Empereur, qu'il la luy eut peut-estre accordé, beaucoup de Ministres estant d'avis dans ce temps là que cette Province ayant tousjours eu ses Princes particuliers, l'Empereur ne la conserveroit pas sans luy donner un Prince et que ne pouvant ny en mettre de plus attaché à sa couronne que Son Altesse Sa Majesté devoit la luy donner ${ }^{35}$.

Cependant, la réponse du duc à cette initiative fut catégorique :

Cependant il ne voulut pas le faire ny mesme donner croyance à ce qu'on luy en escrivit, il estimoit au contraire que ce qu'on luy en mandoit n'estoit que pour l'animer à une chose qu'il avoit répugnance de faire, car sans examiner les raisons de

33. Albert Sorel, L'Europe et la Révolution française, t. I, Les moeurs politiques et les traditions, Paris, Plon, 1885 , p. 447-448.

34. Ce document vient d'être publié en français par les soins du professeur Jean Bérenger : Cardinal Léopold Kollonich, Le plan de réorganisation de la Hongrie (1688), Das Einrichtungswerk des Königreichs Ungarn, Paris, Honoré Champion, 2011.

35. ÖStA, Haus-, Hof- und Staatsarchiv, Hausarchiv, Lothringisches Hausarchiv, Kt. 74, $\mathrm{n}^{\circ}$ 1, Extrait des registres, t. 2, ff. 55-56. 
la Cour il avoit peine d'estre l'instrument pour déposséder le Prince Abaffi contre la parole qu'on luy avoit donné de le maintenir d'ailleurs il craignoit que s'il pretendoit la Transilvanie on ne luy proposa de traitter de la Lorraine et ne voulant pas céder ses droits certains pour une prétention incertaine ny l'espoir de restablir ses enfans dans l'heritage de ses ancestres, pays catholique et le meilleur de l'Europe pour les placer dans le voisinage des Turcs dans une province meslée de toutes sortes de religions et au milieu d'un peuple barbare il ne respondit rien du tout à ceux qui luy en parlèrent. Ils escrivit meme au comte Ferari de n'en rien tesmoigner de sa part ny à l'Empereur ny à personne ${ }^{36}$.

Le Journal nous donne des renseignements très riches sur la manière de combattre à la hongroise, la fameuse petite guerre à la hussarde, ainsi que sur la création et l'emploi des premiers régiments de hussards au service de l'armée impériale. La révolte d'Émeric Thököly mit déjà en relief la capacité de la cavalerie légère hongroise face à l'armée régulière impériale. L'armée impériale ne cesse d'utiliser les services importants des hussards hongrois et se met à tenter une régularisation du corps des hussards en régiments. Après le succès de la cavalerie légère hongroise dans les guerres turques et dans la guerre de Trente ans, la première tentative fut le régiment des soldats hongrois des confins, constitué sous le nom de régiment de cavalerie hongroise sous la direction de François Barkóczy ${ }^{37}$ en 1672/1673. Durant la guerre de la reconquête sur les Turcs, et surtout après 1683, déjà trois régiments de hussards (Barkóczy, Gombos ${ }^{38}$ et Petneházy ${ }^{39}$ ) combattaient dans l'armée impériale. Malgré leurs succès, l'évaluation de ces régiments au sein de l'armée impériale n'était pas encore tout à fait positive. Ils se trouvaient souvent à la fin des listes des régiments ou bien on les mentionnait dans les revues des troupes comme des unités fort différentes et très particulières. Après la libération du château de Bude, un quatrième régiment nommé régiment de cavalerie croate a été fondé sous la direction de Jean Horváth. Ces unités ont été successivement réformées entre 1688 et 1692 . D'autres unités irrégulières combattaient toujours dans l'armée impériale, la plus nombreuse était celle du comte Adam Czobor qui enrôla environ trois milles hommes durant les campagnes de 1686-1687. Le commandement général de l'armée engagea le comte à monter deux régiments de hussards pour opérer dans le Palatinat. La lettre patente (datée du 10 décembre 1688) de l'empereur Léopold évoque ainsi le besoin de monter deux régiments de cavalerie sur le territoire du Royaume de Hongrie composés de cavaliers de nationalité hongroise ! Le comte Czobor commanda le premier régiment jusqu'à sa mort survenue en 1690, puis le régiment changea de nom et devint Kollonits. Le deuxième, fut le régiment Pálffy dans un premier temps, jusqu'en 1700, date à laquelle le comte Ladislas Ebergényi remplaça Pálffy. Le succès de ces régiments dans la guerre en Palatinat favorisa la création d'autres pour les opérations militaires dans la continuation de la reconquête hongroise. Déjà un certain nombre de régiments irréguliers (Gyürky, Bercsényi, Batthyány, Zichy, Bottyán etc.) furent levés dans les années 1692-1693. Ces unités largement décimées au cours de la guerre furent ensuite incorporés dans d'autres régiments de cavalerie. En 1696, l'empereur Léopold nomma l'illustre Paul Deák au rang de colonel et le chargea de former un régiment de cavalerie hongroise. À ce régiment nouvellement créé se joignirent les deux autres

36. Ibid., fol. 56 .

37. François Barkóczy (v. 1627-1709), comte de, colonel puis vice-général impérial et général au service du prince François II Rákóczi.

38. Émeric Gombos (?- ?), colonel de hussards.

39. David Petneházy (v. 1645-1686/1687), capitaine, ensuite colonel dans l'armée de Thököly, puis colonel au service impérial. 
transférés de l'Ouest sur le territoire hongrois. Durant la guerre, ils ont bien mérité les compliments d'Eugène de Savoie. Après la signature du traité de Karlowitz, les régiments devaient être réformés jusqu'à la guerre de Succession d'Espagne ${ }^{40}$.

Notons ici que des unités de cavaliers hongrois furent déjà employées dans l'armée de Charles IV de Lorraine durant la guerre de Trente Ans. L'arrivée des cavaliers légers hongrois en Rhénanie en 1635 fut un événement exceptionnel, comme en témoigne une anecdote racontée par l'historien Dom Calmet : «Là un Régiment de Hongrois joignit Son Altesse, \& à son passage tailla en pièces la Garnison du Château de Hambourg. Le Duc Charles eut beaucoup de peine de tirer de leurs mains un Capitaine qu'ils vouloient sacrifier sur le bord du Rhin, en perpétuelle mémoire de leur passage de ce fleuve ; étant les premiers de leur Nation, qui depuis plusieurs siècles s'étoient fait voir en deça du Rhin. ${ }^{41}$ Charles IV de Lorraine mit très rapidement sa confiance dans les Hongrois dont il composa son escorte lorsqu'il traversa une région truffée de Français pour rencontrer le général Gallas en octobre $1635^{42}$.

D'après son Journal, le duc Charles V de Lorraine employa environ 2500 cavaliers hongrois en Rhénanie au cours de la campagne de 1689. Ils y furent très utiles dans la reconnaissance des mouvements ennemis ainsi que dans les opérations de petite guerre. Par ailleurs, le duc de Lorraine reconnut l'importance d'autres troupes légères levées en Allemagne dont il compléta son armée. Les opérations des fameux « chenapans ${ }^{43}$ furent souvent évoquées dans le journal de la campagne de cette année. Certaines autres méthodes empruntées aux campagnes précédentes y furent employées avec succès. Quelques astuces de la guerre turque semblèrent être très utiles contre les Français lors du siège de Mayence. Quoique les arcs et les flèches ne fussent plus des armes utilisées dans les armées occidentales de la fin du XVII ${ }^{\mathrm{e}}$ siècle, ils remplirent une tâche importante dans une guerre psychologique pour réduire le moral des défenseurs :

Le Duc qui avoit eu cet avis d'ailleurs voulant tascher d'affoiblir la garnison par la desertion aussy bien que par le feu s'avisa de faire jetter dans la place des billets à des fleches en la maniere que les Tartares attachent les feux d'artifice pour bruler les villes. Ces billets portoient que les generaux de l'Empereur et de ses alliez ayant esté avertys que les soldats de la garnison de Mayence apprehendoient d'en sortir crainte d'estre mal traittez dans le camp, il les assuroit qu'ils y trouveroient toute sorte de seureté et de bon traittement, soit qu'ils voulussent prendre service, soit qu'ils voulussent passer ailleurs ${ }^{44}$.

Au terme de cette présentation, le Journal des campagnes de Charles de Lorraine nous apparaît comme une source primordiale sur le début de la guerre de reconquête de la Hongrie sur les Turcs. Au-delà des problèmes de l'identification de l'auteur de cet ouvrage,

40. Ferenc Tóth, « La diffusion des hussards en Europe, $\mathrm{XV}^{\mathrm{e}}$ siècle-XIX ${ }^{\mathrm{e}}$ siècle », Cahiers d'études et de recherches du musée de l'Armée, 2005-2006/6 (Armes et cultures de guerre en Europe centrale XVII ${ }^{\mathrm{e}}$ siècle-XVIII $^{\mathrm{e}}$ siècle), p. 226-227.

41. Dom Augustin Calmet, Histoire ecclésiastique et civile de la Lorraine, t. III, Nancy, 1728, p. 306.

42. Jean-Charles Fulaine, Le Duc Charles IV de Lorraine et son armée 1624-1675, Metz, Éd. Serpenoise, 1997, p. 129.

43. Chenapan, maradeur (aussi snapane, snaphaine « maradeur » au XVII ${ }^{\mathrm{e}}$ siècle), le mot vient très probablement de l'allemand Schapphahn signifiant « arquebuse ».

44. ÖStA, Haus-, Hof- und Staatsarchiv, Hausarchiv, Lothringisches Hausarchiv, Kt. 51, $\mathrm{n}^{\circ}$ 3, Beschreibung der Feldzüge Herzogs Karl V. von Lothringen (1683-89), fol. 323. 
nous pouvons constater qu'il présente une chronique très précise et bien détaillée des événements des neuf premières années de la guerre turque. Les comparaisons avec les autres sources de contrôle, aussi bien les sources d'archives que les mémoires et relations publiés, nous prouvent qu'il s'agit d'une relation particulièrement fiable des opérations militaires commentée par des remarques politiques justes. Paradoxalement, les historiens autrichiens et hongrois, n'ont pas accordé une grande importance à cette source, ni non plus à l'ouvrage de Dom Calmet (Histoire ecclésiastique et civile de Lorraine) qui en tira des informations essentielles relatives aux années 1683-1689, car l'un et l'autre étaient souvent considérés comme des textes relatifs à l'histoire des ducs de Lorraine, voire à l'histoire d'une région française. L'histoire de la vie de Charles V de Lorraine, le « duc sans duchés », ne s'intégrait que bien difficilement dans les cadres des histoires nationales à partir du XIX ${ }^{\mathrm{e}}$ siècle, ce qui explique en partie cet oubli. Très probablement, des recherches historiques et philologiques en vue de la publication du Journal permettront bientôt d'avoir plus de renseignements sur cette source importante. 